\title{
ON THE CONSTANT IN THE NONUNIFORM VERSION OF THE BERRY-ESSEEN THEOREM
}

\author{
K. NEAMMANEE
}

Received 24 November 2004 and in revised form 8 March 2005

Dedicated to Professor Yupaporn Kemprasit on her sixtieth birthday.

In 2001, Chen and Shao gave the nonuniform estimation of the rate of convergence in Berry-Esseen theorem for independent random variables via Stein-Chen-Shao method. The aim of this paper is to obtain a constant in Chen-Shao theorem, where the random variables are not necessarily identically distributed and the existence of their third moments are not assumed. The bound is given in terms of truncated moments and the constant obtained is 21.44 for most values. We use a technique called Stein's method, in particular the Chen-Shao concentration inequality.

\section{Introduction and main result}

Let $X_{1}, X_{2}, \ldots, X_{n}$ be independent and not necessarily identically distributed random variables with zero mean and finite variance. Define $W=X_{1}+X_{2}+\cdots+X_{n}$ and assume that $\operatorname{Var}(W)=1$. Let $F_{n}$ be the distribution function of $W$ and $\Phi$ the standard normal distribution function. It is well known that if the Lindeberg condition,

$$
\forall \varepsilon>0, \quad \sum_{i=1}^{n} E X_{i}^{2} I\left(\left|X_{i}\right|>\varepsilon\right) \longrightarrow 0 \quad \text { as } n \longrightarrow \infty,
$$

where $I(A)$ is an indicator random variable such that

$$
I(A)= \begin{cases}1 & \text { if } A \text { is true } \\ 0 & \text { otherwise }\end{cases}
$$

is satisfied, then

$$
\forall x \in \mathbb{R}, \quad F_{n}(x) \longrightarrow \Phi(x) \quad \text { as } n \longrightarrow \infty
$$

Furthermore, if $E\left|X_{i}\right|^{3}<\infty$, then we have the uniform Berry-Esseen theorem

$$
\sup _{x \in \mathbb{R}}\left|F_{n}(x)-\Phi(x)\right| \leq C_{0} \sum_{i=1}^{n} E\left|X_{i}\right|^{3},
$$


and the nonuniform Berry-Esseen theorem

$$
\left|F_{n}(x)-\Phi(x)\right| \leq \frac{C_{1}}{(1+|x|)^{3}} \sum_{i=1}^{n} E\left|X_{i}\right|^{3}
$$

where both $C_{0}$ and $C_{1}$ are absolute constants.

Note that in case $X_{i}$ 's are identically distributed, (1.4) and (1.5) were first obtained by Esseen [4] and Nagaev [8], respectively. Bikjalis [1] generalized Nagaev's result to the case that $X_{i}$ 's are not necessarily identically distributed random variables. Paditz $[9,10]$ calculated $C_{1}$ to be 114.7 and 32 in 1977 and 1989, respectively, and Michel [7] reduced it to 30.84 for the independent and identically distributed case.

In 2001, Chen and Shao gave nonuniform and uniform bounds for independent and not necessarily identically distributed random variables without assuming the existence of third moments. Their result states as follows.

Theorem 1.1 (Chen-Shao theorem). Let $X_{1}, X_{2}, \ldots, X_{n}$ be independent random variables with zero means and $\sum_{i=1}^{n} E X_{i}^{2}=1$. Let $W=X_{1}+X_{2}+\cdots+X_{n}$ and let $F_{n}$ be the distribution function of $W$. Then,

$$
\begin{gathered}
|F(x)-\Phi(x)| \leq C \sum_{i=1}^{n}\left\{\frac{E X_{i}^{2} I\left(\left|X_{i}\right| \geq 1+|x|\right)}{(1+|x|)^{2}}+\frac{E\left|X_{i}\right|^{3} I\left(\left|X_{i}\right|<1+|x|\right)}{(1+|x|)^{3}}\right\}, \\
|F(x)-\Phi(x)| \leq 4.1 \sum_{i=1}^{n}\left\{E X_{i}^{2} I\left(\left|X_{i}\right| \geq 1\right)+E\left|X_{i}\right|^{3} I\left(\left|X_{i}\right|<1\right)\right\} .
\end{gathered}
$$

Observe that the constant 4.1 in (1.7) is smaller than 6 as obtained by Feller [5] and it was pointed out by Loh [6] that the truncation at 1 in (1.7) is optimal in the sense that

$$
E X^{2} I(|X| \geq 1)+E|X|^{3} I(|X|<1)=\inf _{A}\left\{E X^{2} I(X \in A)+E|X|^{3} I\left(X \in A^{C}\right)\right\} .
$$

The standard tool used Esseen [4], Nagaev [8], Bikjalis [1], Paditz [9, 10], and Michel [7] is the Fourier-analytic method. But Chen and Shao [3] proved (1.6) and (1.7) by combining truncation with Stein's method [14] and the concentration inequality approach. The concentration inequality approach was originally used by Stein for independent and identically distributed random variables. It was extended by Chen [2] to dependent and nonidentically distributed random variables with arbitrary index sets. In [3], the concentration inequality approach is improved and extended to nonuniform bounds. The improved approach is much more effective than that in [2]. In this paper, we combine the concentration inequality in [3] with the coupling approach to calculate the constant $C$ in (1.6). The followings are our main results.

Theorem 1.2. Let $X_{1}, X_{2}, \ldots, X_{n}$ be independent random variables with zero means and $\sum_{i=1}^{n} E X_{i}^{2}=1$. Let $W=X_{1}+X_{2}+\cdots+X_{n}$ and let $F_{n}$ be the distribution function of $W$. Then

$$
\left|F_{n}(x)-\Phi(x)\right| \leq C_{0} \sum_{i=1}^{n}\left\{\frac{E X_{i}^{2} I\left(\left|X_{i}\right| \geq 1+|x / 4|\right)}{(1+|x / 4|)^{2}}+\frac{E\left|X_{i}\right|^{3} I\left(\left|X_{i}\right|<1+|x / 4|\right)}{(1+|x / 4|)^{3}}\right\},
$$


where

$$
C_{0}= \begin{cases}21.44 & \text { if }|x| \leq 3 \text { or }|x| \geq 14 \\ 32 & \text { if } 3<|x| \leq 3.99 \text { or } 7.98<|x|<14 \\ 60 & \text { otherwise. }\end{cases}
$$

Corollary 1.3. If $X_{i}$ 's in Theorem 1.1 have finite third moment, then

$$
\left|F_{n}(x)-\Phi(x)\right| \leq \frac{C_{1} \sum_{i=1}^{n} E\left|X_{i}\right|^{3}}{(1+|x / 4|)^{3}},
$$

where

$$
C_{1}= \begin{cases}21.44 & \text { if }|x| \leq 7.98 \text { or }|x| \geq 14 \\ 32 & \text { if } 7.98<|x|<14\end{cases}
$$

Observe that the bound in Theorem 1.2 is given in terms of truncated moments. It is worthwhile to note also that truncated moments were considered by Sazonov [13]. In his work, he gave two main methods for deriving speed of convergence results in the central limit theorem (CLT), namely, the Fourier-analytic method and the method of composition which used convolutions directly. These methods are used to derive more results for random vectors. For nonuniform bound in CLT of random vectors, one can see, for examples, Rotar $[11,12]$.

\section{Auxiliary results}

In this section, we give auxiliary results in order to prove the main theorem in Section 3. Let $X_{1}, X_{2}, \ldots, X_{n}, W, F_{n}$, and $\Phi$ be defined as in Theorem 1.2. In order to use the concentration inequality and the coupling approach, we introduce random variables $J, \tilde{X}_{1}$, $\tilde{X}_{2}, \ldots, \tilde{X}_{n}$ defined in the following way. The random variables $J, X_{1}, X_{2}, \ldots, X_{n}, \tilde{X}_{1}$, $\tilde{X}_{2}, \ldots, \tilde{X}_{n}$ are independent, $J$ uniformly distributed over the set $\{1,2, \ldots, n\},\left(X_{i}, \tilde{X}_{i}\right)$ is a coupling pair, that is, $X_{i}$ and $\tilde{X}_{i}$ are the same distributions. For $a>0$, we also let

$$
\begin{gathered}
Y_{j, a}=X_{j} I\left(\left|X_{j}\right|<1+a\right), \quad \tilde{Y}_{j, a}=\tilde{X}_{j} I\left(\left|\tilde{X}_{j}\right|<1+a\right), \\
S_{a}=\sum_{j=1}^{n} Y_{j, a}, \quad \tilde{S}_{a}=S_{a}-Y_{J, a}+\tilde{Y}_{J, a}, \\
\alpha_{a}=\sum_{j=1}^{n} E X_{j}^{2} I\left(\left|X_{j}\right| \geq 1+a\right), \quad \beta_{a}=\sum_{j=1}^{n} E\left|X_{j}\right|^{3} I\left(\left|X_{j}\right|<1+a\right), \\
\delta_{a}=\frac{\alpha_{a}}{(1+a)^{2}}+\frac{\beta_{a}}{(1+a)^{3}} .
\end{gathered}
$$

Observe that $\left(Y_{j, a}, \tilde{Y}_{j, a}\right)$ is a coupling pair and $\left(S_{a}, \widetilde{S}_{a}\right)$ is an exchangeable pair in the sense that

$$
P\left(S_{a} \in E, \widetilde{S}_{a} \in \widetilde{E}\right)=P\left(S_{a} \in \widetilde{E}, \widetilde{S}_{a} \in E\right)
$$




\section{On the constant of the Berry-Esseen theorem}

for arbitrary Borel sets $E$ and $\tilde{E}$ on $\mathbb{R}$. From the fact that $(a+b)^{n} \leq 2^{n-1}\left(a^{n}+b^{n}\right)$ for $a, b \geq 0$, we have

$$
\begin{gathered}
E\left|Y_{J, a}\right|^{3}=\frac{1}{n} \sum_{j=1}^{n} E\left|X_{j}\right|^{3} I\left(\left|X_{j}\right|<1+a\right)=\frac{\beta_{a}}{n}, \\
E\left|\tilde{Y}_{J, a}-Y_{J, a}\right|^{3} \leq \frac{8}{n} \sum_{j=1}^{n} E\left|X_{j}\right|^{3} I\left(\left|X_{j}\right|<1+a\right)=\frac{8 \beta_{a}}{n} .
\end{gathered}
$$

In proposition 2.1, we use the coupling approach to bound $E S_{a}^{2}$ and $E S_{a}^{4}$ which are used in the proof of the concentration inequality.

Proposition 2.1. (1) $E^{S_{a}} \tilde{S}_{a}=(1-1 / n) S_{a}+(1 / n) E S_{a}$, where $E^{X} Y$ is the conditional expectation of $Y$ with respect to $X$.

(2) $E S_{a}^{2} \leq 1+\left(\alpha_{a} /(1+a)\right)^{2}$.

(3) $\left|E S_{a}\right|^{3} \leq 12 \beta_{a}+3\left(\alpha_{a} /(1+a)\right)+\left(\alpha_{a} /(1+a)\right)^{3}$.

(4) $E S_{a}^{4} \leq 53(1+a) \beta_{a}+30 \beta_{a}\left(\alpha_{a} /(1+a)\right)+6\left(\alpha_{a} /(1+a)\right)^{2}+\left(\alpha_{a} /(1+a)\right)^{4}+6 \beta_{a}+$ $6 \alpha_{a}+3$.

(5) If $(1+a)^{2} \alpha_{a}+(1+a) \beta_{a}<1 / 80$ and $a \geq 3$, then $E S_{a}^{2} \leq 1+\left(3.8 \times 10^{-8}\right)$ and $E S_{a}^{4} \leq$ 3.69 .

(6) If $(1+a)^{2} \alpha_{a}+(1+a) \beta_{a} \geq 1 / 80$ and $a \geq 14$, then $E_{a}^{4} / a^{4} \leq 391 \delta_{a}$.

Proof. (1)

$$
\begin{aligned}
E^{S_{a} \tilde{S}_{a}} & =E^{S_{a}}\left(S_{a}-Y_{J, a}+\tilde{Y}_{J, a}\right) \\
& =S_{a}-E^{S_{a}} Y_{J, a}+E^{S_{a}} \tilde{Y}_{J, a} \\
& =S_{a}-\frac{1}{n} \sum_{j=1}^{n} E^{S_{a}} Y_{j, a}+\frac{1}{n} \sum_{j=1}^{n} E^{S_{a}} \tilde{Y}_{j, a} \\
& =S_{a}-\frac{1}{n} \sum_{j=1}^{n} Y_{j, a}+\frac{1}{n} \sum_{j=1}^{n} E Y_{j, a} \\
& =\left(1-\frac{1}{n}\right) S_{a}+\frac{1}{n} E S_{a} .
\end{aligned}
$$

(2) Let $h: \mathbb{R}^{2} \rightarrow \mathbb{R}$ be defined by

$$
h(\tilde{t}, t)=\tilde{t}^{2}-t^{2} .
$$

Since $h$ is antisymmetric in the sense that $h(\tilde{t}, t)=-h(t, \tilde{t})$ and $\left(S_{a}, \tilde{S}_{a}\right)$ is an exchangeable pair, by Stein [15, equation (9), page 10],

$$
E\left(\tilde{S}_{a}^{2}-S_{a}^{2}\right)=E h\left(\tilde{S}_{a}, S_{a}\right)=0 .
$$


From this fact and (1), we have

$$
\begin{aligned}
0 & =E\left(\tilde{S}_{a}-S_{a}\right)\left(\tilde{S}_{a}+S_{a}\right) \\
& =2 E\left(\tilde{S}_{a}-S_{a}\right) S_{a}+E\left(\tilde{S}_{a}-S_{a}\right)^{2} \\
& =2 E\left(E^{S_{a}} \tilde{S}_{a}-S_{a}\right) S_{a}+E\left(\tilde{S}_{a}-S_{a}\right)^{2} \\
& =-\frac{2}{n} E S_{a}^{2}+E\left(\tilde{S}_{a}-S_{a}\right)^{2}+\frac{2}{n} E^{2} S_{a},
\end{aligned}
$$

which implies that

$$
\begin{aligned}
E S_{a}^{2} & =\frac{n}{2} E\left(\tilde{Y}_{J, a}-Y_{J, a}\right)^{2}+E^{2} S_{a} \\
& =\sum_{j=1}^{n}\left\{E X_{j}^{2} I\left(\left|X_{j}\right|<1+a\right)-E^{2} X_{j} I\left(\left|X_{j}\right|<1+a\right)\right\}+E^{2} S_{a} \\
& \leq \sum_{j=1}^{n} E X_{j}^{2} I\left(\left|X_{j}\right|<1+a\right)+E^{2} S_{a} \\
& \leq 1+\left(\frac{\alpha_{a}}{1+a}\right)^{2}
\end{aligned}
$$

where we have used the fact that $\sum_{j=1}^{n} E X_{j}^{2}=1$ and

$$
\left|E S_{a}\right|=\left|\sum_{j=1}^{n} E X_{j} I\left(\left|X_{j}\right|<1+a\right)\right|=\left|\sum_{j=1}^{n} E X_{j} I\left(\left|X_{j}\right| \geq 1+a\right)\right| \leq \frac{\alpha_{a}}{1+a}
$$

in the last inequality.

(3) By the same argument of (2), with $h(\tilde{t}, t)=(\tilde{t}-t)\left(\tilde{t}^{2}+t^{2}\right)$,

$$
\begin{aligned}
E S_{a}^{3} & =\frac{n}{2} E\left(\tilde{S}_{a}-S_{a}\right)\left(\tilde{S}_{a}^{2}-S_{a}^{2}\right)+E S_{a} E S_{a}^{2} \\
& =\frac{n}{2} E\left(\tilde{S}_{a}-S_{a}\right)^{2}\left(\tilde{S}_{a}+S_{a}\right)+E S_{a} E S_{a}^{2} \\
& =\frac{n}{2} E\left(\tilde{Y}_{J, a}-Y_{J, a}\right)^{2}\left[\left(\tilde{Y}_{J, a}-Y_{J, a}\right)+2 S_{a}\right]+E S_{a} E S_{a}^{2} \\
& =\frac{n}{2} E\left(\tilde{Y}_{J, a}-Y_{J, a}\right)^{3}+n E\left(\tilde{Y}_{J, a}-Y_{J, a}\right)^{2} S_{a}+E S_{a} E S_{a}^{2}
\end{aligned}
$$

Hence,

$$
\begin{aligned}
\left|E S_{a}^{3}\right| & \leq \frac{n}{2} E\left|\tilde{Y}_{J, a}-Y_{J, a}\right|^{3}+n\left|E\left(\tilde{Y}_{J, a}-Y_{J, a}\right)^{2} S_{a}\right|+E S_{a} E S_{a}^{2} \\
& \leq 4 \beta_{a}+n\left|E\left(\tilde{Y}_{J, a}-Y_{J, a}\right)^{2} S_{a}\right|+\left(\frac{\alpha_{a}}{1+a}\right)+\left(\frac{\alpha_{a}}{1+a}\right)^{3}
\end{aligned}
$$


where we have used (2.4), (2.10), and (2) in the last inequality. Note that

$$
\begin{aligned}
\left|E\left(\tilde{Y}_{J, a}-Y_{J, a}\right)^{2} S_{a}\right|= & \left|\frac{1}{n} \sum_{j=1}^{n} E\left(\tilde{Y}_{j, a}-Y_{j, a}\right)^{2} \sum_{l=1}^{n} Y_{l, a}\right| \\
\leq & \left|\frac{1}{n} \sum_{j=1}^{n} E\left(\tilde{Y}_{j, a}-Y_{j, a}\right)^{2} Y_{j, a}\right|+\left|\frac{1}{n} \sum_{j=1}^{n} E\left(\tilde{Y}_{j, a}-Y_{j, a}\right)^{2} \sum_{\substack{l=1 \\
l \neq j}}^{n} E Y_{l, a}\right| \\
\leq & \frac{1}{n} \sum_{j=1}^{n} E\left(\tilde{Y}_{j, a}-Y_{j, a}\right)^{2}\left|Y_{j, a}\right|+\frac{1}{n} \sum_{j=1}^{n} E\left(\tilde{Y}_{j, a}-Y_{j, a}\right)^{2}\left|E S_{a}\right| \\
& +\frac{1}{n} \sum_{j=1}^{n} E\left(\tilde{Y}_{j, a}-Y_{j, a}\right)^{2} E\left|Y_{j, a}\right| \\
\leq & \frac{8}{n} \sum_{j=1}^{n} E\left|Y_{j, a}\right|^{3}+\frac{2}{n}\left(\frac{\alpha_{a}}{1+a}\right) \\
\leq & \frac{8 \beta_{a}}{n}+\frac{2}{n}\left(\frac{\alpha_{a}}{1+a}\right) .
\end{aligned}
$$

Hence, by (2.12) and (2.13), $\left|E S_{a}^{3}\right| \leq 12 \beta_{a}+3\left(\alpha_{a} /(1+a)\right)+\left(\alpha_{a} /(1+a)\right)^{3}$.

(4) Using the same argument of $(2)$, with $h(\tilde{t}, t)=(\tilde{t}-t)\left(\tilde{t}^{3}+t^{3}\right)$, we have

$$
\begin{aligned}
E S_{a}^{4}= & \frac{n}{2} E\left(\tilde{S}_{a}-S_{a}\right)\left(\tilde{S}_{a}^{3}-S_{a}^{3}\right)+E S_{a} E S_{a}^{3} \\
= & \frac{n}{2} E\left(\tilde{S}_{a}-S_{a}\right)^{2}\left[\left(\tilde{S}_{a}-S_{a}\right)^{2}+3 \tilde{S}_{a} S_{a}\right]+E S_{a} E S_{a}^{3} \\
= & \frac{n}{2} E\left(\tilde{Y}_{J, a}-Y_{J, a}\right)^{4}+\frac{3 n}{2} E\left(\tilde{Y}_{J, a}-Y_{J, a}\right)^{2}\left(S_{a}^{2}+\left(\tilde{Y}_{J, a}-Y_{J, a}\right) S_{a}\right)+E S_{a} E S_{a}^{3} \\
\leq & n(1+a) E\left|\tilde{Y}_{J, a}-Y_{J, a}\right|^{3}+\frac{3 n}{2} E\left(\tilde{Y}_{J, a}-Y_{J, a}\right)^{2} S_{a}^{2} \\
& +3 n(1+a) E\left|\left(\tilde{Y}_{J, a}-Y_{J, a}\right)^{2} S_{a}\right|+E S_{a} E S_{a}^{3} \\
\leq & 32(1+a) \beta_{a}+6 \alpha_{a}+12 \beta_{a}\left(\frac{\alpha_{a}}{1+a}\right)+3\left(\frac{\alpha_{a}}{1+a}\right)^{2}+\left(\frac{\alpha_{a}}{1+a}\right)^{4} \\
& +\frac{3 n}{2} E\left(\tilde{Y}_{J, a}-Y_{J, a}\right)^{2} S_{a}^{2}
\end{aligned}
$$

where we have used (2.4), (2.10), (2.13), and (3) in the last inequality. From (2.14) and the fact that

$$
\begin{aligned}
& E\left(\tilde{Y}_{J, a}-Y_{J, a}\right)^{2} S_{a}^{2} \\
& \quad=\frac{1}{n} \sum_{j=1}^{n} E\left(\tilde{Y}_{j, a}-Y_{j, a}\right)^{2} E\left(\sum_{\substack{l=1 \\
l \neq j}}^{n} Y_{l, a}\right)^{2}+\frac{2}{n} \sum_{j=1}^{n} E\left(\tilde{Y}_{j, a}-Y_{j, a}\right)^{2} Y_{j, a} E\left(\sum_{\substack{l=1 \\
l \neq j}}^{n} Y_{l, a}\right)
\end{aligned}
$$




$$
\begin{aligned}
& +\frac{1}{n} \sum_{j=1}^{n} E\left(\tilde{Y}_{j, a}-Y_{j, a}\right)^{2} Y_{j, a}^{2} \\
\leq & \frac{2}{n} \sum_{j=1}^{n} E Y_{j, a}^{2} E\left(\sum_{\substack{l=1 \\
l \neq j}}^{n} Y_{l, a}\right)^{2}+\frac{8}{n} \sum_{j=1}^{n} E\left|Y_{j, a}\right|^{3}\left|E\left(\sum_{\substack{l=1 \\
l \neq j}}^{n} Y_{l, a}\right)\right|+\frac{4(1+a)}{n} \sum_{j=1}^{n} E\left|Y_{j, a}\right|^{3} \\
\leq & \frac{2}{n} \sum_{j=1}^{n} E Y_{j, a}^{2} E S_{a}^{2}-\frac{4}{n} \sum_{j=1}^{n} E Y_{j, a}^{2} E S_{a} Y_{j, a}+\frac{2}{n} \sum_{j=1}^{n} E Y_{j, a}^{2} E Y_{j, a}^{2} \\
& +\frac{8}{n} \sum_{j=1}^{n} E\left|Y_{j, a}\right|^{3}\left|E S_{a}\right|+\frac{8}{n} \sum_{j=1}^{n} E\left|Y_{j, a}\right|^{3}\left|E Y_{j, a}\right|+\frac{4(1+a)}{n} \beta_{a} \\
\leq & \frac{2}{n}+\frac{2}{n}\left(\frac{\alpha_{a}}{1+a}\right)^{2}+\frac{4}{n} \sum_{j=1}^{n} E\left|Y_{j, a}\right|^{3} \sqrt{E S_{a}^{2}}+\frac{8 \beta_{a}}{n}\left(\frac{\alpha_{a}}{1+a}\right)+\frac{14(1+a) \beta_{a}}{n} \\
\leq & \frac{2}{n}+\frac{4 \beta_{a}}{n}+\frac{12 \beta_{a}}{n}\left(\frac{\alpha_{a}}{1+a}\right)+\frac{2}{n}\left(\frac{\alpha_{a}}{1+a}\right)^{2}+\frac{14(1+a) \beta_{a}}{n},
\end{aligned}
$$

we have

$$
E S_{a}^{4} \leq 53(1+a) \beta_{a}+30 \beta_{a}\left(\frac{\alpha_{a}}{1+a}\right)+6\left(\frac{\alpha_{a}}{1+a}\right)^{2}+\left(\frac{\alpha_{a}}{1+a}\right)^{4}+6 \beta_{a}+6 \alpha_{a}+3
$$

(5) Follows directly from (2) and (4).

(6)

$$
\begin{aligned}
\frac{E S_{a}^{4}}{a^{4}} \leq & \frac{53}{a^{3}}\left(\frac{1+a}{a}\right) \beta_{a}+\frac{30 \beta_{a}}{a^{4}}\left(\frac{\alpha_{a}}{1+a}\right)+\frac{6}{a^{4}}\left(\frac{\alpha_{a}}{1+a}\right)^{2} \\
& +\frac{1}{a^{4}}\left(\frac{\alpha_{a}}{1+a}\right)^{4}+\frac{6 \beta_{a}}{a^{4}}+\frac{6 \alpha_{a}}{a^{4}}+\frac{3}{a^{4}} \\
\leq & \frac{70.697 \beta_{a}}{(1+a)^{3}}+\frac{0.035 \alpha_{a}}{(1+a)^{2}}+\frac{3.997}{(1+a)^{4}} \\
\leq & \frac{70.697 \beta_{a}}{(1+a)^{3}}+\frac{0.035 \alpha_{a}}{(1+a)^{2}}+319.76 \alpha_{a} \\
\leq & 391 \delta_{a},
\end{aligned}
$$

where we have used the fact that $a \geq 14, \alpha_{a} \leq 1$, and $(1+a) / a \leq 1.072$ in the second inequality and the fact that $(1+a)^{2} \alpha_{a}+(1+a) \beta_{a} \geq 1 / 80$ in the last inequality.

Next, we will prove the concentration inequality.

Proposition 2.2 (concentration inequality). Let $i \in\{1,2, \ldots, n\}$ and $W^{(i)}=W-X_{i}$. Then for $3 \leq a<b<\infty$ and $(1+a)^{2} \alpha_{a}+(1+a) \beta_{a}<1 / 80$,

$$
P\left(a \leq W^{(i)} \leq b\right) \leq \frac{40.98}{(1+a)^{3}}(b-a)+46.38 \delta_{a} .
$$


1958 On the constant of the Berry-Esseen theorem

Proof. Let $S_{i, a}=S_{a}-Y_{i, a}$. We observe that $W^{(i)}=S_{i, a}$ when $\max _{1 \leq j \leq n, j \neq i}\left|X_{j}\right|<1+a$. So

$$
\begin{aligned}
P\left(a \leq W^{(i)} \leq b\right) & \leq P\left(a \leq S_{i, a} \leq b\right)+P\left(\max _{\substack{1 \leq j \leq n \\
j \neq i}}\left|X_{j}\right| \geq 1+a\right) \\
& \leq P\left(a \leq S_{i, a} \leq b\right)+\frac{\alpha_{a}}{(1+a)^{2}} .
\end{aligned}
$$

Let $\gamma=\beta_{a} / 2$ and $f: \mathbb{R} \rightarrow \mathbb{R}$ defined by

$$
f(t)= \begin{cases}0 & \text { for } t<a-\gamma \\ (1+t+\gamma)^{3}(t-a+\gamma) & \text { for } a-\gamma \leq t \leq b+\gamma \\ (1+t+\gamma)^{3}(b-a+2 \gamma) & \text { for } t>b+\gamma\end{cases}
$$

So $f$ is a nondecreasing function satisfying $f^{\prime}(t) \geq(1+a)^{3}$ for $a-\gamma<t<b+\gamma$, and $f^{\prime}(t) \geq 0$ otherwise. Let $M(w, t)=w[I(-w \leq t<0)-I(0 \leq t<-w)]$. Hence,

$$
\begin{aligned}
& E S_{i, a} f\left(S_{i, a}\right)=\sum_{\substack{j=1 \\
j \neq i}}^{n} E Y_{j, a}\left(f\left(S_{i, a}\right)-f\left(S_{i, a}-Y_{j, a}\right)\right) \\
& =\sum_{\substack{j=1 \\
j \neq i}}^{n} E Y_{j, a} \int_{-Y_{j, a}}^{0} f^{\prime}\left(S_{i, a}+t\right) d t \\
& =\sum_{\substack{j=1 \\
j \neq i}}^{n} E Y_{j, a}\left\{\int_{\mathbb{R}} f^{\prime}\left(S_{i, a}+t\right)\left[I\left(-Y_{j, a} \leq t<0\right)-I\left(0<t \leq-Y_{j, a}\right)\right] d t\right\} \\
& =\sum_{\substack{j=1 \\
j \neq i}}^{n} E\left\{\int_{\mathbb{R}} f^{\prime}\left(S_{i, a}+t\right) M\left(Y_{j, a}, t\right) d t\right\} \\
& \geq(1+a)^{3} \sum_{\substack{j=1 \\
j \neq i}}^{n} E\left\{I\left(a \leq S_{i, a} \leq b\right) \int_{|t| \leq \gamma} M\left(Y_{j, a}, t\right) d t\right\} \\
& =(1+a)^{3} E\left\{I\left(a \leq S_{i, a} \leq b\right) \sum_{\substack{j=1 \\
j \neq i}}^{n}\left|Y_{j, a}\right| \min \left(\gamma,\left|Y_{j, a}\right|\right)\right\} \\
& \geq 0.46(1+a)^{3}\left\{P\left(a \leq S_{i, a} \leq b\right)-P\left(U_{i} \leq 0.46\right)\right\} \text {, }
\end{aligned}
$$

where $U_{i}=\sum_{j=1, j \neq i}^{n}\left|Y_{j, a}\right| \min \left(\gamma,\left|Y_{j, a}\right|\right)$ and we have used the fact that

$$
I\left(t_{1} \leq w \leq t_{2}\right) y \geq c\left(I\left(t_{1} \leq w \leq t_{2}\right)-\left(1-\frac{y}{c}\right) I(y \leq c)\right)
$$


for $t_{1}, t_{2}, y \geq 0, c>0$ in the last inequality. Hence,

$$
P\left(a \leq S_{i, a} \leq b\right) \leq \frac{1}{0.46(1+a)^{3}} E S_{i, a} f\left(S_{i, a}\right)+P\left(U_{i} \leq 0.46\right) .
$$

Next, we will bound the two terms on the right-hand side of (2.23). By the same argument as that in Proposition 2.1, we can show that $E S_{i, a}^{4} \leq 3.69$ and $E S_{i, a}^{2} \leq 1+\left(3.8 \times 10^{-8}\right)$. So

$$
\begin{aligned}
E\left|S_{i, a} f\left(S_{i, a}\right)\right| & \leq(b-a+2 \gamma) E\left|S_{i, a}\right|\left|S_{i, a}+(1+\gamma)\right|^{3} \\
& \leq 4\left(b-a+\beta_{a}\right)\left(E S_{i, a}^{4}+|1+\gamma|^{3} E\left|S_{i, a}\right|\right) \\
& \leq 4\left(b-a+\beta_{a}\right)\left(E S_{i, a}^{4}+\left|1+\frac{\beta_{a}}{2}\right|^{3} \sqrt{E S_{i, a}^{2}}\right) \\
& \leq 18.85\left(b-a+\beta_{a}\right) .
\end{aligned}
$$

By the facts that $\min (a, b) \geq b-b^{2} / 4 a$ for $a, b>0$,

$$
\begin{gathered}
E X_{i}^{2} I\left(\left|X_{i}\right| \leq 1+a\right) \leq\left(\beta_{a}\right)^{2 / 3}<0.021, \\
\alpha_{a} \leq \frac{1}{80(1+a)^{2}} \leq 7.8 \times 10^{-4} \text { for } a \geq 3,
\end{gathered}
$$

we have

$$
\begin{aligned}
E U_{i} & =\sum_{\substack{j=1 \\
j \neq i}}^{n} E\left|Y_{j, a}\right| \min \left(\gamma,\left|Y_{j, a}\right|\right) \\
\geq & \sum_{\substack{j=1 \\
j \neq i}}^{n}\left(E Y_{j, a}^{2}-\frac{E\left|Y_{j, a}\right|^{3}}{4 \gamma}\right) \\
\geq & \sum_{\substack{j=1 \\
n}}^{n}\left\{E X_{j}^{2} I\left(\left|X_{j}\right|<1+a\right)-E X_{j}^{2} I\left(\left|X_{j}\right| \geq 1+a\right)\right\}-\frac{\beta}{\gamma} \\
& =\sum_{\substack{j=1 \\
j \neq i}}\left\{E X_{j}^{2} I\left(\left|X_{j}\right|<1+a\right)-E^{2} X_{j} I\left(\left|X_{j}\right| \geq 1+a\right)\right\}-0.5 \\
& =1-E X_{i}^{2} I\left(\left|X_{i}\right|<1+a\right)-2 \sum_{j=1}^{n} E X_{j}^{2} I\left(\left|X_{i}\right| \geq 1+a\right)-0.5 \\
\geq & 1-\left(\beta_{a}\right)^{2 / 3}-2 \alpha_{a}-0.5 \\
\geq & 0.477 .
\end{aligned}
$$

Using the same argument as in Proposition 2.1(5), we can show that

$$
E\left|U_{i}-E U_{i}\right|^{4} \leq 3.69 \gamma^{4}=0.231 \beta_{a}^{4} \leq 4.512 \times 10^{-7} \frac{\beta_{a}}{(1+a)^{3}} .
$$


1960 On the constant of the Berry-Esseen theorem

Hence,

$$
\begin{aligned}
P\left(U_{i} \leq 0.46\right) & \leq P\left(E U_{i}-U_{i} \geq 0.477-0.46\right) \\
& =P\left(E U_{i}-U_{i} \geq 0.017\right) \\
& \leq \frac{E\left|U_{i}-E U_{i}\right|^{4}}{(0.017)^{4}} \\
& \leq \frac{5.402 \beta_{a}}{(1+a)^{3}}
\end{aligned}
$$

From (2.19), (2.23), (2.24), and (2.28),

$$
\begin{aligned}
P\left(a \leq W^{(i)} \leq b\right) & \leq \frac{40.978}{(1+a)^{3}}\left(b-a+\beta_{a}\right)+\frac{5.402 \beta_{a}}{(1+a)^{3}}+\frac{\alpha_{a}}{(1+a)^{2}} \\
& \leq \frac{40.98(b-a)}{(1+a)^{3}}+46.36 \delta_{a} .
\end{aligned}
$$

Proposition 2.3. For $x \geq 2$,

$$
E\left|f_{x}^{\prime}(W)\right| \leq \frac{15}{(1+x)^{2}}
$$

where $f_{x}$ is the unique solution of the Stein equation

$$
f^{\prime}(w)-w f(w)=I(w \leq x)-\Phi(x)
$$

Proof. From Stein [15, pages 22 and 24], we know that

$$
\begin{gathered}
0<f_{x}^{\prime}(w)<1-\Phi(x) \quad \text { for } w \leq 0, \\
0<f_{x}^{\prime}(x) \leq 1-\Phi(x)\left[1+\sqrt{2 \pi} w e^{(1 / 2) w^{2}} \Phi(x)\right] \quad \text { for } 0<w \leq x, \\
\left|f_{x}^{\prime}(w)\right| \leq 1 \quad \forall w \in \mathbb{R} .
\end{gathered}
$$

Hence,

$$
\begin{aligned}
E\left|f_{x}^{\prime}(W)\right|= & E\left|f_{x}^{\prime}(W)\right| I(W \leq 0)+E\left|f_{x}^{\prime}(W)\right| I\left(0<W \leq \frac{4 x}{5}\right) \\
& +E\left|f_{x}^{\prime}(W)\right| I\left(W>\frac{4 x}{5}\right) \\
\leq & (1-\Phi(x)) P(W \leq 0)+(1-\Phi(x)) E\left(1+\sqrt{2 \pi} W e^{W^{2} / 2}\right) I\left(0<W \leq \frac{4 x}{5}\right) \\
& +\frac{E\left(1+W^{2}\right)}{(1+(4 x / 5))^{2}} \\
\leq & (1-\Phi(x))+(1-\Phi(x))\left(1+\frac{4 \sqrt{2 \pi}}{5} x e^{8 x^{2} / 25}\right)+\frac{2}{(1+(4 x / 5))^{2}} .
\end{aligned}
$$


Since

$$
1-\Phi(x) \leq \frac{e^{-(1 / 2) x^{2}}}{\sqrt{2 \pi} x} \quad \text { for } x \geq 0
$$

(see Stein [15, equation (25), page 23]) and $e^{x^{2} / 2}>x$ for $x \geq 2$, we have

$$
(1-\Phi(x))(1+x)^{2} \leq \frac{1}{\sqrt{2 \pi} x^{2}}(1+x)^{2}=\frac{1}{\sqrt{2 \pi}}\left(\frac{1}{x}+1\right)^{2} \leq 0.9,
$$

which implies that

$$
1-\Phi(x) \leq \frac{0.9}{(1+x)^{2}}
$$

From (2.34) and the fact that $e^{9 x^{2} / 50}>9 x^{2} / 50$, we derive

$$
\begin{aligned}
\sqrt{2 \pi}(1-\Phi(x))(1+x)^{2} x e^{8 x^{2} / 25} & \leq e^{-9 x^{2} / 50}(1+x)^{2} \\
& \leq \frac{50}{9}\left(\frac{1}{x}+1\right)^{2} \\
& \leq 12.5
\end{aligned}
$$

that is,

$$
\frac{4 \sqrt{2 \pi}}{5}(1-\Phi(x)) x e^{8 x^{2} / 25} \leq \frac{10}{(1+x)^{2}} .
$$

From (2.33), (2.36), (2.38), and the fact that $(1+x) /(1+4 x / 5) \leq 5 / 4$, we have proved the proposition.

Proposition 2.4. Let $x \geq 14$ and $g: \mathbb{R} \rightarrow \mathbb{R}$ defined by $g(w)=\left(w f_{x}(w)\right)^{\prime}$. If $(1+x)^{2} \alpha_{x}+$ $(1+x) \beta_{x}<1 / 80$, then for $|u| \leq 1+x / 4$,

$$
E g\left(W^{(i)}+u\right) \leq \frac{4.60}{(1+x / 4)^{3}}+5.13 \delta_{x / 4}(1+x) .
$$

Proof. From Chen and Shao [3, pages 248-249], we know that

$$
g(x-1)=\left[\sqrt{2 \pi}\left(1+(x-1)^{2}\right) e^{(x-1)^{2} / 2} \Phi(x-1)+(x-1)\right](1-\Phi(x)),
$$

$g$ is increasing for $0 \leq w<x$, and

$$
E g\left(W^{(i)}+u\right) \leq \frac{2}{1+x^{3}}+2(1-\Phi(x))+g(x-1)+E g\left(W^{(i)}+u\right) I\left(x-1<W^{(i)}+u<x\right) .
$$

For $x \geq 14$, elementary calculation yields

$$
\frac{(1+x)^{3}}{1+x^{3}} \leq 1.23
$$


1962 On the constant of the Berry-Esseen theorem

and $e^{x^{2} / 2} \geq(1 / 3 !)\left(x^{2} / 2\right)^{3} \geq 800 x^{2}$. Using similar argument as that in deriving (2.36), we have

$$
1-\Phi(x) \leq \frac{0.0006}{(1+x)^{3}}
$$

By (2.40) and (2.34),

$$
\begin{aligned}
g(x-1) & \leq \sqrt{2 \pi}\left(1+(x-1)^{2}\right) e^{(x-1)^{2} / 2}(1-\Phi(x))+x(1-\Phi(x)) \\
& \leq\left(x-2+\frac{2}{x}\right) e^{-x+1 / 2}+\frac{1}{\sqrt{2 \pi}} e^{-(1 / 2) x^{2}} \\
& \leq \frac{0.056}{(1+x)^{3}}
\end{aligned}
$$

where we have used the fact that $f(x)=\left\{(x-2+2 / x) e^{-x+1 / 2}+(1 / \sqrt{2 \pi}) e^{-(1 / 2) x^{2}}\right\}(1+x)^{3}$ is decreasing on $[14, \infty)$ in the last inequality. So, by $(2.41),(2.42),(2.43)$, and (2.44),

$$
\begin{aligned}
E g\left(W^{(i)}+u\right) \leq & \frac{2.517}{(1+x)^{3}}+E g\left(W^{(i)}+u\right) I\left(x-1<W^{(i)}+u<x\right) \\
= & \frac{2.517}{(1+x)^{3}}+\int_{x-1}^{x}-g(w) d P\left(w<W^{(i)}+u<x\right) \\
= & \frac{2.517}{(1+x)^{3}}+g(x-1) P\left(x-1<W^{(i)}+u<x\right) \\
& +\int_{x-1}^{x} g^{\prime}(w) P\left(w<W^{(i)}+u<x\right) d w \\
\leq & \frac{2.573}{(1+x)^{3}}+\int_{x-1}^{x} g^{\prime}(w)\left[\frac{40.98}{(1+w-u)^{3}}(x-w)+46.38 \delta_{w-u}\right] d w
\end{aligned}
$$

where the last inequality follows from Proposition 2.2, (2.44), and the fact that

$$
w-u \geq(x-1)-\left(1+\frac{x}{4}\right) \geq \frac{3 x}{5} \geq 3 \text { for }|u|<1+\frac{x}{4} .
$$

Since $\delta_{x}$ is decreasing in $x, g$ is nonnegative and increasing on $[0, x)$, and $|g(x)| \leq 1+|x|$, (2.45) can then be bounded by

$$
\begin{aligned}
E g\left(W^{(i)}+u\right) & \leq \frac{2.573}{(1+x)^{3}}+\frac{40.98}{(1+3 x / 5)^{3}} \int_{x-1}^{x} g^{\prime}(w)(x-w) d w+46.38 \delta_{3 x / 5} g(x) \\
& \leq \frac{2.573}{(1+x)^{3}}+\frac{40.98}{(1+3 x / 5)^{3}} \int_{x-1}^{x}(x-w) d g(w)+46.38(1+x) \delta_{3 x / 5} \\
& \leq \frac{2.573}{(1+x)^{3}}+\frac{40.98}{(1+3 x / 5)^{3}} \int_{x-1}^{x} g(w) d w+46.38(1+x) \delta_{3 x / 5} \\
& =\frac{2.573}{(1+x)^{3}}+\frac{40.98}{(1+3 x / 5)^{3}}\left(x f_{x}(x)\right)+46.38(1+x) \delta_{3 x / 5} \\
& \leq \frac{4.60}{(1+x / 4)^{3}}+5.13 \delta_{x / 4}(1+x),
\end{aligned}
$$


where we have applied the fact that $\left|x f_{x}(x)\right| \leq 1,(1+x / 4) /(1+x) \leq 0.30,(1+x / 4) /(1+$ $3 x / 5) \leq 0.48$, and $\delta_{3 x / 5} \leq(1+x / 4)^{3} /(1+3 x / 5)^{3} \delta_{x / 4} \leq 0.111 \delta_{x / 4}$ for $x \geq 14$ in the last inequality.

We are now ready to prove our main results.

\section{Proof of main results}

3.1. Proof of Theorem 1.2. It suffices to consider only $x \geq 0$ as we can simply apply the result to $-W$ when $x<0$.

Case $3.1(0 \leq x \leq 3)$. By (1.7) and (1.8),

$$
\begin{aligned}
21.44 & \sum_{i=1}^{n}\left[\frac{E X_{i}^{2} I\left(\left|X_{i}\right| \geq 1+x / 4\right)}{(1+x / 4)^{2}}+\frac{E\left|X_{i}\right|^{3} I\left(\left|X_{i}\right|<1+x / 4\right)}{(1+x / 4)^{3}}\right] \\
& \geq 4.1 \sum_{i=1}^{n}\left[E X_{i}^{2} I\left(\left|X_{i}\right| \geq 1+\frac{x}{4}\right)+E\left|X_{i}\right|^{3} I\left(\left|X_{i}\right|<1+\frac{x}{4}\right)\right] \\
& \geq 4.1 \sum_{i=1}^{n}\left[E X_{i}^{2} I\left(\left|X_{i}\right| \geq 1\right)+E\left|X_{i}\right|^{3} I\left(\left|X_{i}\right|<1\right)\right] \\
& \geq|F(x)-\Phi(x)| .
\end{aligned}
$$

Case $3.2(x \geq 14)$.

Subcase $3.3\left((1+x)^{2} \alpha_{x}+(1+x) \beta_{x} \geq 1 / 80\right)$. Using similar argument as that in showing (2.36), we see that $1-\Phi(x) \leq 0.009 /(1+x)^{4}$. This inequality, together with inequality $(1+x)^{2} \alpha_{x}+(1+x) \beta_{x} \geq 1 / 80$, gives $1-\Phi(x) \leq 0.738 \delta_{x}$, which in turn implies that

$$
\begin{aligned}
|F(x)-\Phi(x)| & \leq P(W \geq x)+(1-\Phi(x)) \\
& \leq P(W \geq x)+0.738 \delta_{x} .
\end{aligned}
$$

Using the same argument as in (2.19), we have

$$
\begin{aligned}
P(W \geq x) & \leq P\left(S_{x} \geq x\right)+\frac{\alpha_{x}}{(1+x)^{2}} \\
& \leq \frac{E S_{x}^{4}}{x^{4}}+\frac{\alpha_{x}}{(1+x)^{2}} \quad \text { (which by Proposition 2.1(6)) } \\
& \leq 391 \delta_{a}+\frac{\alpha_{x}}{(1+x)^{2}} \\
& \leq 392 \delta_{x} .
\end{aligned}
$$

From (3.2), (3.3), and the fact that $\delta_{x} \leq((1+x / 4) /(1+x))^{3} \delta_{x / 4} \leq 0.05 \delta_{x / 4}$, we have

$$
|F(x)-\Phi(x)| \leq 20.26 \delta_{x / 4}
$$


1964 On the constant of the Berry-Esseen theorem

Subcase $3.4\left((1+x)^{2} \alpha_{x}+(1+x) \beta_{x}<1 / 80\right)$. Let $K_{i, x / 4}(t)=E Y_{i, x / 4}\left\{I\left(0<t \leq Y_{i, x / 4}\right)\right.$ $\left.-I\left(Y_{i, x / 4} \leq t<0\right)\right\}$. From Chen and Shao [3, pages 250-251], we set

$$
F(x)-\Phi(x)=R_{1}+R_{2}+R_{3}+R_{4},
$$

where

$$
\begin{gathered}
R_{1}=\sum_{i=1}^{n} E\left\{I\left(\left|X_{i}\right|<1+\frac{x}{4}\right) \int_{|t| \leq 1+x / 4}\left(f_{x}^{\prime}\left(W^{(i)}+X_{i}\right)-f_{x}^{\prime}\left(W^{(i)}+t\right)\right) K_{i, x / 4}(t) d t\right\} \\
R_{2}=\sum_{i=1}^{n} E\left\{I\left(\left|X_{i}\right| \geq 1+\frac{x}{4}\right) \int_{|t| \leq 1+x / 4}\left(f_{x}^{\prime}\left(W^{(i)}+X_{i}\right)-f_{x}^{\prime}\left(W^{(i)}+t\right)\right) K_{i x / 4}(t) d t\right\} \\
R_{3}=\alpha_{x / 4} E f_{x}^{\prime}(W), \\
R_{4}=-\sum_{i=1}^{n} E\left\{X_{i} I\left(\left|X_{i}\right| \geq 1+\frac{x}{4}\right)\left(f(W)-f\left(W^{(i)}\right)\right)\right\}
\end{gathered}
$$

and observe that $\left|R_{1}\right| \leq R_{11}+R_{12}$, where

$$
\begin{gathered}
R_{11}=\sum_{i=1}^{n}\left|E\left\{I\left(\left|X_{i}\right|<1+\frac{x}{4}\right) \int_{|t| \leq 1+x / 4} K_{i, x / 4}(t) \int_{t}^{X_{i}} E g\left(W^{(i)}+u\right) d u d t\right\}\right|, \\
R_{12}=\sum_{i=1}^{n} E\left\{I ( | X _ { i } | < 1 + \frac { x } { 4 } ) \int _ { | t | \leq 1 + x / 4 } P \left(x-\max \left(t, X_{i}\right) \leq W^{(i)} \leq x\right.\right. \\
\left.\left.-\min \left(t, X_{i}\right) \mid X_{i}\right) K_{i, x / 4}(t) d t\right\} .
\end{gathered}
$$

By the fact that $\left|f_{x}^{\prime}(s)-f_{x}^{\prime}(t)\right| \leq 1$ for all $x, s, t \in \mathbb{R}$ (see Chen and Shao [3, page 246]), we have

$$
\begin{aligned}
\left|R_{2}\right| & \leq \sum_{i=1}^{n} P\left(\left|X_{i}\right| \geq 1+\frac{x}{4}\right) \\
& \leq \sum_{i=1}^{n} \frac{E X_{i}^{2} I\left(\left|X_{i}\right| \geq 1+x / 4\right)}{(1+x / 4)^{2}} \\
& =\frac{\alpha_{x / 4}}{(1+x / 4)^{2}}
\end{aligned}
$$

and by Proposition 2.3,

$$
\left|R_{3}\right|=\alpha_{x / 4}\left|E f_{x}^{\prime}(W)\right| \leq \frac{15 \alpha_{x / 4}}{(1+x)^{2}} \leq \frac{15 \alpha_{x / 4}}{(1+x / 4)^{2}} .
$$


Since $0 \leq f_{x}(w) \leq \min (\sqrt{2 \pi} / 4,1 /|x|)$ for all $x>0$ (see Chen and Shao [3, equation (4.6), page 246]),

$$
\begin{aligned}
\left|R_{4}\right| & \leq \sum_{i=1}^{n} \frac{E\left|X_{i}\right| I\left(\left|X_{i}\right| \geq 1+x / 4\right)}{x} \\
& \leq \sum_{i=1}^{n} \frac{E X_{i}^{2} I\left(\left|X_{i}\right| \geq 1+x / 4\right)}{(1+x / 4) x} \\
& \leq \frac{\alpha_{x / 4}}{(1+x / 4) x} \\
& \leq \frac{\alpha_{x / 4}}{(1+x / 4)^{2}} .
\end{aligned}
$$

Hence,

$$
\left|R_{2}+R_{3}+R_{4}\right| \leq \frac{17 \alpha_{x / 4}}{(1+x / 4)^{2}}
$$

By Proposition 2.4, we have

$$
\begin{aligned}
R_{11} & \leq 2\left[\frac{4.60}{(1+x / 4)^{3}}+5.13(1+x) \delta_{x / 4}\right] \sum_{i=1}^{n} E\left|Y_{i, x / 4}\right|^{3} \\
& \leq \frac{9.2 \beta_{x / 4}}{(1+x / 4)^{3}}+10.26(1+x) \delta_{x / 4} \beta_{x / 4} \\
& \leq \frac{9.2 \beta_{x / 4}}{(1+x / 4)^{3}}+0.128 \delta_{x / 4},
\end{aligned}
$$

where we have used the fact that $(1+x) \beta_{x / 4} \leq(1+x) \beta_{x}<1 / 80$ in the last inequality.

By Proposition 2.2 and the fact that

$$
x-\max \left(t, X_{i}\right) \geq x-\left(1+\frac{x}{4}\right)=\frac{3 x}{4}-1 \geq \frac{2 x}{3} \quad \text { for }|t| \leq 1+\frac{x}{4},
$$

we have

$$
\begin{aligned}
\left|R_{12}\right| & \leq \sum_{i=1}^{n} E\left\{I\left(\left|X_{i}\right| \leq 1+\frac{x}{4}\right) \int_{|t| \leq 1+x / 4}\left(\frac{40.98}{(1+2 x / 3)^{3}}\left(|t|+\left|X_{i}\right|\right)+46.38 \delta_{2 x / 3}\right) K_{i, x / 4}(t) d t\right\} \\
& \leq \frac{81.96}{(1+2 x / 3)^{3}} \beta_{x / 4}+46.38 \delta_{2 x / 3} \\
& \leq \frac{6.982}{(1+x / 4)^{3}} \beta_{x / 4}+3.942 \delta_{x / 4}
\end{aligned}
$$

where we have used the fact that $(1+x / 4) /(1+2 x / 3) \leq 0.44$ and $\delta_{2 x / 3} \leq(1+x / 4)^{3} /(1$ $+2 x / 3)^{3} \delta_{x / 4} \leq 0.085 \delta_{x / 4}$ for $x \geq 14$ in the last inequality. 
1966 On the constant of the Berry-Esseen theorem

Hence, by (3.5), (3.11), (3.12), and (3.14),

$$
|F(x)-\Phi(x)| \leq\left|R_{1}+R_{2}+R_{3}+R_{4}\right| \leq 20.26 \delta_{x / 4} .
$$

For the case when $3<x<14$, we can use the same arguments as in Case 3.1 and Case 3.2.

3.2. Proof of Corollary 1.3. From van Beek [16], we know that

$$
|F(x)-\Phi(x)| \leq 0.7975 \sum_{i=1}^{n} E\left|X_{i}\right|^{3}
$$

Using the same technique in proving Case 3.1 of Theorem 1.2, we see that

$$
|F(x)-\Phi(x)| \leq 21.44 \sum_{i=1}^{n} E\left|X_{i}\right|^{3}
$$

for $0 \leq x \leq 7.98$. For $x>7.98$, we use Theorem 1.1 and the fact that

$$
\frac{E X_{i}^{2} I\left(\left|X_{i}\right| \geq 1+|x / 4|\right)}{(1+|x / 4|)^{2}}+\frac{E\left|X_{i}\right|^{3} I\left(\left|X_{i}\right|<1+|x / 4|\right)}{(1+|x / 4|)^{3}} \leq \frac{E\left|X_{i}\right|^{3}}{(1+|x / 4|)^{3}} .
$$

\section{Remark}

In the proof of Theorem 1.1, we assume that $x \geq a$, where $a=14$. It is not necessary to assume that $a=14$. If $a>14$ is used, the constant will be sharper.

\section{Acknowledgments}

The author would like to thank the referees for useful comments and acknowledge Thailand Research Fund for a financial support.

\section{References}

[1] A. Bikjalis, Estimates of the remainder term in the central limit theorem, Litovsk. Mat. Sb. 6 (1966), no. 3, 323-346.

[2] L. H. Y. Chen, The Rate of Convergence in a Central Limit Theorem for Dependent Random Variables with Arbitrary Index Set, IMA Preprint Series \#243, University of Minnesota, Minnesota, 1986.

[3] L. H. Y. Chen and Q.-M. Shao, A non-uniform Berry-Esseen bound via Stein's method, Probab. Theory Related Fields 120 (2001), no. 2, 236-254.

[4] C.-G. Esseen, Fourier analysis of distribution functions. A mathematical study of the LaplaceGaussian law, Acta Math. 77 (1945), 1-125.

[5] W. Feller, On the Berry-Esseen theorem, Z. Wahrsch. Verw. Gebiete 10 (1968), 261-268.

[6] W. Y. Loh, On the normal approximation for sums of mixing random variables, Master thesis, Department of Mathematics, University of Singapore, Singapore, 1975.

[7] R. Michel, On the constant in the nonuniform version of the Berry-Esseen theorem, Z. Wahrsch. Verw. Gebiete 55 (1981), no. 1, 109-117.

[8] S. V. Nagaev, Some limit theorems for large deviations, Theory Probab. Appl. 10 (1965), 214235. 
[9] L. Paditz, Über die Annäherung der Verteilungsfunktionen von Summen unabhängiger Zufallsgrößen gegen unbegrenzt teilbare Verteilungsfunktionen unter besonderer beachtung der Verteilungsfunktion der standardisierten Normalverteilung, Dissertation A., Technische Universität Dresden, Dresden, 1977.

[10] On the analytical structure of the constant in the nonuniform version of the Esseen inequality, Statistics 20 (1989), no. 3, 453-464.

[11] V. I. Rotar, Non classical estimates of the rate of convergence in the multidimensional central limit theorem. I, Teor. Veroyatnost. i Primenen. 22 (1977), no. 7, 774-790 (Russian).

[12] Non classical estimates of the rate of convergence in the multidimensional central limit theorem II, Teor. Veroyatnost. i Primenen. 23 (1978), no. 1, 55-66 (Russian).

[13] V. V. Sazonov, Normal Approximation-Some Recent Advances, Lecture Notes in Mathematics, vol. 879, Springer, Berlin, 1981.

[14] C. Stein, A bound for the error in the normal approximation to the distribution of a sum of dependent random variables, Proceedings of the 6th Berkeley Symposium on Mathematical Statistics and Probability (Univ. California, Berkeley, Calif, 1970/1971), Vol. II: Probability Theory, University of California Press, California, 1972, pp. 583-602.

[15] Approximate Computation of Expectations, Institute of Mathematical Statistics Lecture Notes-Monograph Series, 7, Institute of Mathematical Statistics, California, 1986.

[16] P. van Beek, An application of Fourier methods to the problem of sharpening the Berry-Esseen inequality, Z. Wahrsch. Verw. Gebiete 23 (1972), 187-196.

K. Neammanee: Department of Mathematics, Faculty of Science, Chulalongkorn University, Bangkok 10330, Thailand

E-mail address: kritsana.n@chula.ac.th 


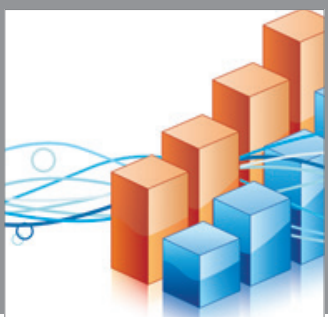

Advances in

Operations Research

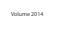

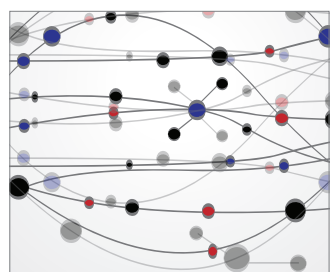

\section{The Scientific} World Journal
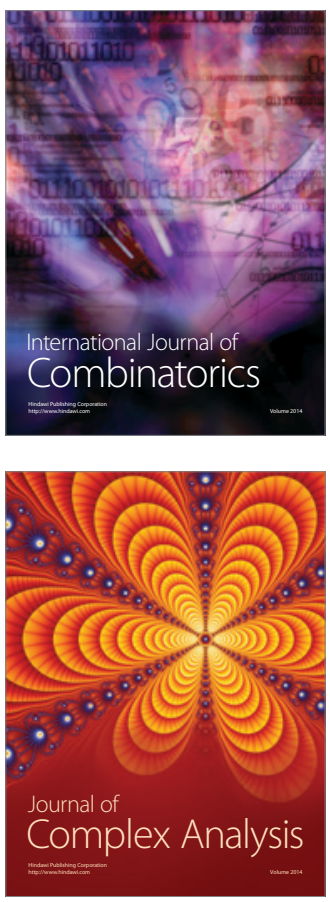

International Journal of

Mathematics and

Mathematical

Sciences
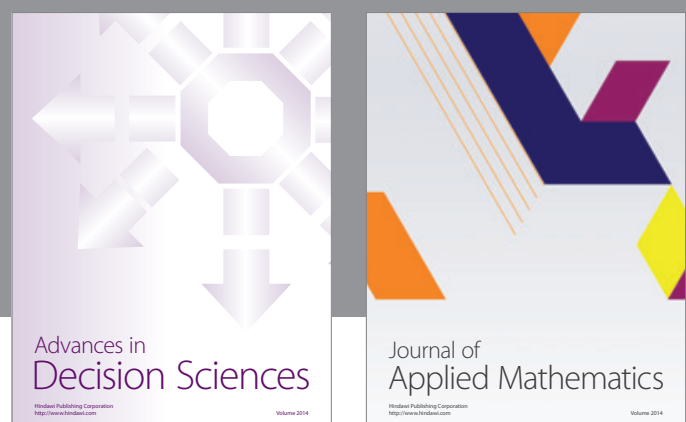

Journal of

Applied Mathematics
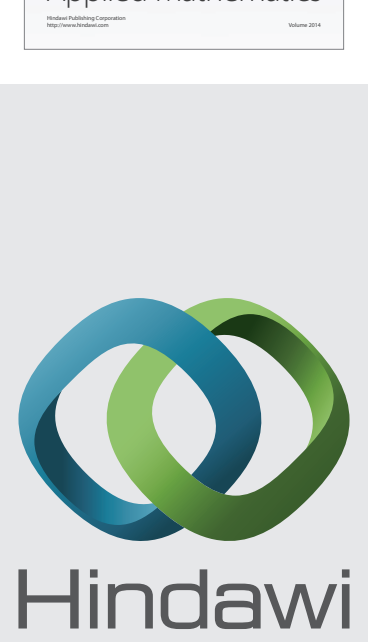

Submit your manuscripts at http://www.hindawi.com
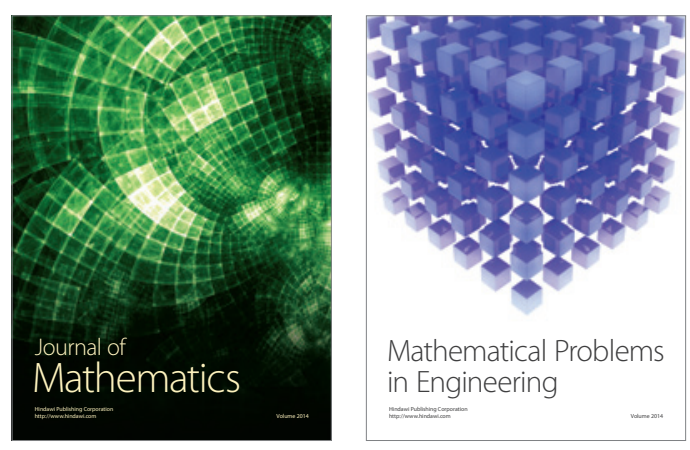

Mathematical Problems in Engineering
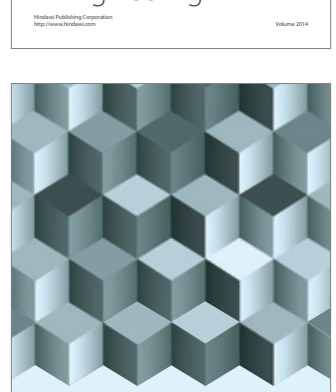

Journal of

Function Spaces
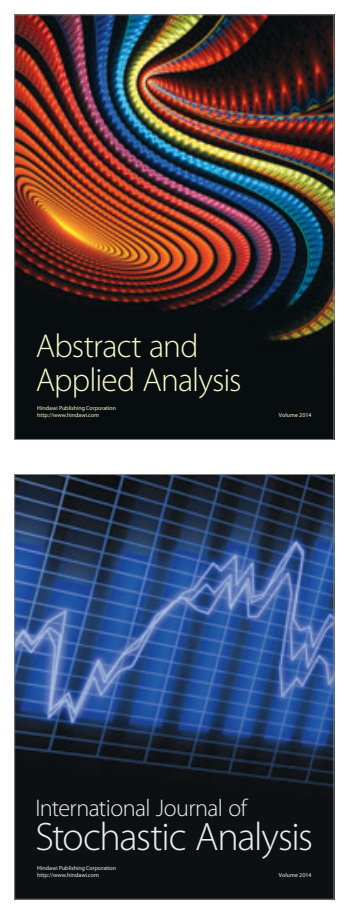

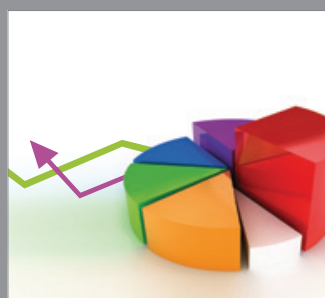

ournal of

Probability and Statistics

Promensencen
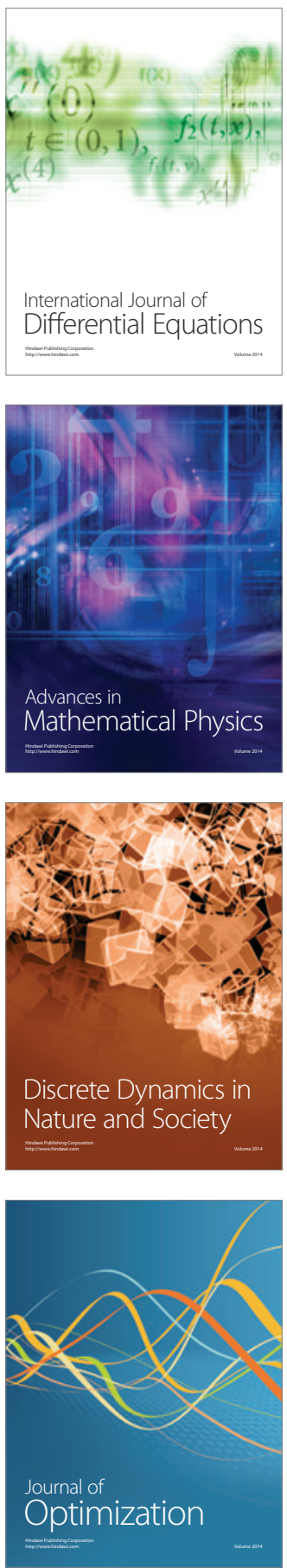